\title{
On approximate solution of Drygas functional equation according to the Lipschitz criteria
}

\author{
Iz-iddine EL-Fassi \\ Department of Mathematics, Faculty of Sciences, \\ Ibn Tofail University, B.P. 133, Kenitra, Morocco \\ email: izidd-math@hotmail.fr; \\ izelfassi.math@gmail.com
}

\begin{abstract}
Let $\mathrm{G}$ be an Abelian group with a metric $\mathrm{d}$ and $\mathrm{E}$ be a normed space. For any $f: G \rightarrow E$ we define the Drygas difference of the function $f$ by the formula

$$
\Lambda f(x, y):=2 f(x)+f(y)+f(-y)-f(x+y)-f(x-y)
$$

for all $x, y \in G$. In this article, we prove that if $\Lambda f$ is Lipschitz, then there exists a Drygas function $D: G \rightarrow E$ such that $f-D$ is Lipschitz with the same constant. Moreover, some results concerning the approximation of the Drygas functional equation in the Lipschitz norms are presented.
\end{abstract}

\section{Introduction}

The stability theory of functional equations began with the well-known Ulam's Problem [21], concerning the stability of homomorphisms in metric groups:

Problem. Let $\left(\mathrm{G}_{1}, *\right),\left(\mathrm{G}_{2}, \star\right)$ be two groups and $\mathrm{d}: \mathrm{G}_{2} \times \mathrm{G}_{2} \rightarrow[0, \infty)$ be a metric. Given $\epsilon>0$, does there exist $\delta>0$ such that if a function $f: G_{1} \rightarrow G_{2}$ satisfies the inequality

$$
d(f(x * y), f(x) \star f(y)) \leq \delta
$$

2010 Mathematics Subject Classification: 65Q20, 39B82, 41A65

Key words and phrases: Drygas functional equation, stability, Lipschitz space 
for all $x, y \in G_{1}$, then there is a homomorphism $h: G_{1} \rightarrow G_{2}$ with

$$
d(f(x), h(x)) \leq \epsilon
$$

for all $x \in \mathrm{G}_{1}$ ?

Ulam's problem was partially solved by Hyers [14] in 1941 in the context of Banach spaces with $\delta=\epsilon$. Aoki [1], Z. Gajda [11] and Th.M. Rassias [17] provided a generalization of the result of Hyers for additive and linear mappings, respectively, by allowing the Cauchy difference to be unbounded. Since then many authors have studied the question of stability of various functional equations (see $[9,15]$ for the survey of stability results).

Let $G$ and $Y$ be an Abelian group and a Banach space respectively. We say that a function $f: G \rightarrow Y$ satisfies the Drygas equation if

$$
f(x+y)+f(x-y)=2 f(x)+f(y)+f(-y), \quad x, y \in G,
$$

and every solution of the Drygas equation is called a Drygas function. The above equation was introduced in [4] to obtain a characterization of the quasiinner-product spaces. The general solution of (1), obtained by Ebanks et al. in [5] (see also[18]). The stability in the Hyers-Ulam sense of the Drygas equation has been investigated, for example, in $[6,7,10,16,19,22]$.

In Lipschitz spaces the stability type problems for some functional equations was studied by a number of mathematicians (see, e.g., [3, 8, 20])

In the present paper, we establish the stability problem of (1) in Lipschitz spaces.

\section{Preliminaries}

In this section we are going to introduce some basic definitions and notations needed for further considerations.

Definition 1 [2] Let $\mathbb{R}$ be the set of real numbers, E a vector space and $\mathcal{S}(\mathrm{E})$ a family of subsets of $\mathrm{E}$. We say that this family is linearly invariant if

(1) $x+\alpha \mathrm{V} \in \mathrm{S}(\mathrm{E})$ for $x \in \mathrm{E}, \alpha \in \mathbb{R}$ and $\mathrm{V} \in \mathcal{S}(\mathrm{E})$,

(2) $\mathrm{V}+\mathrm{W} \in \mathcal{S}(\mathrm{E})$ for $\mathrm{V}, \mathrm{W} \in \mathcal{S}(\mathrm{E})$.

Definition 2 Let $\mathrm{G}$ be a set, $\mathrm{E}$ a vector space and $\mathcal{S}(\mathrm{E})$ any linearly invariant family. By $\mathcal{B}(\mathrm{G}, \mathcal{S}(\mathrm{E}))$ we denote the family

$$
\mathcal{B}(\mathrm{G}, \mathcal{S}(\mathrm{E})):=\{\mathrm{f}: \mathrm{G} \rightarrow \mathrm{E} ; \mathrm{Im} \mathrm{f} \subset \mathrm{V} \text { for some } \mathrm{V} \in \mathcal{S}(\mathrm{E})\} .
$$


It is easy to verify that $\mathcal{B}(\mathrm{G}, \mathcal{S}(\mathrm{E}))$ is a vector space. For any $\mathrm{f}: \mathrm{G} \rightarrow \mathrm{E}$, $\mathrm{a} \in \mathrm{G}$, where $\mathrm{G}$ is a group, we put

$$
f^{a}(x):=f(x+a), \quad x \in G .
$$

Definition $3[13,20]$ Let $\mathrm{G}$ be a group, $\mathrm{E}$ a vector space, and let $\mathcal{S}(\mathrm{E})$ be a linearly invariant family of subsets of $\mathrm{E}$. We say that $\mathcal{B}(\mathrm{G}, \mathcal{S}(\mathrm{E}))$ admits a left invariant mean (LIM for short) if there exists a linear operator $M$ : $\mathcal{B}(\mathrm{G}, \mathcal{S}(\mathrm{E})) \rightarrow \mathrm{E}$ such that

(i) if $\operatorname{Im} \mathrm{f} \subset \mathrm{V}$ for some $\mathrm{V} \in \mathcal{S}(\mathrm{E})$, then $\mathrm{M}[\mathrm{f}] \in \mathrm{V}$,

(ii) if $\mathrm{f} \in \mathcal{B}(\mathrm{G}, \mathcal{S}(\mathrm{E}))$ and $\mathrm{a} \in \mathrm{G}$, then $\mathrm{M}\left[\mathrm{f}^{\mathrm{a}}\right]=\mathrm{M}[\mathrm{f}]$.

Analogously we can define so-called right invariant mean. For more information about spaces which admit LIM see, e.g., [2, 12, 13].

Example 1 Let $\mathrm{G}$ be a finite group, let $\mathrm{E}$ be a vector space, and let $\mathcal{S}(\mathrm{E})$ be any linearly invariant family of convex subsets of $\mathrm{E}$. Let $\mathrm{f} \in \mathcal{B}(\mathrm{G}, \mathcal{S}(\mathrm{E})$ ) be arbitrary. We define

$$
M[f]:=\frac{1}{|G|} \sum_{g \in G} f(g) .
$$

One can easily check that $\mathrm{M}$ is a LIM on $\mathcal{B}(\mathrm{G}, \mathcal{S}(\mathrm{E}))$, where $|\mathrm{G}|=$ cardinality of $\mathrm{G}$.

Definition 4 Let $\mathrm{G}$ be a group, $\mathrm{E}$ a vector space and let $\mathcal{S}(\mathrm{E})$ be a linearly invariant family. We say that $\mathbf{d}: \mathrm{G} \times \mathrm{G} \rightarrow \mathcal{S}(\mathrm{E})$ is translation invariant if

$$
\mathbf{d}(x+a, y+a)=\mathbf{d}(a+x, a+y)=\mathbf{d}(x, y), \text { for all } x, y, a \in G \text {. }
$$

The function $\mathrm{f}: \mathrm{G} \rightarrow \mathrm{E}$ is $\mathbf{d}$-Lipschitz if for all $\mathrm{x}, \mathrm{y} \in \mathrm{G}$,

$$
f(x)-f(y) \in d(x, y) .
$$

Definition 5 Let $\mathrm{G}$ be a group with a metric d and $\mathrm{E}$ a normed space.

a/ We say that $w: \mathbb{R}_{+} \rightarrow \mathbb{R}_{+}$is the module of continuity of $\mathrm{f}: \mathrm{G} \rightarrow \mathrm{E}$ if for every $\delta \in \mathbb{R}_{+}$

$$
\mathrm{d}(x, y) \leq \delta \Rightarrow\|f(x)-f(y)\| \leq w(\delta) \quad x, y \in G .
$$


$\mathrm{b} /$ A function $\mathrm{f}: \mathrm{G} \rightarrow \mathrm{E}$ called a Lipschitz function if there exists an $\mathrm{L} \in \mathbb{R}_{+}$ such that

$$
\|f(x)-f(y)\| \leq \operatorname{Ld}(x, y), \quad x, y \in G .
$$

The smallest constant $\mathrm{L}$ with this property is denoted by $\operatorname{lip}(\mathrm{f})$. By $\operatorname{Lip}(\mathrm{G}, \mathrm{E})$ we mean the space of all bounded Lipschitz functions with the norm

$$
\|\mathbf{f}\|_{\text {Lip }}:=\|\mathbf{f}\|_{\text {sup }}+\operatorname{lip}(\mathbf{f}) .
$$

Moreover, by $\operatorname{Lip}^{0}(\mathrm{G}, \mathrm{E})$ we denote the space of all Lipschitz functions $\mathrm{f}: \mathrm{G} \rightarrow$ $\mathrm{E}$ with the norm defined by the formula

$$
\|\mathbf{f}\|_{L i p^{0}}:=\|\mathbf{f}(0)\|+\operatorname{lip}(\mathbf{f}) .
$$

Finally, we introduce the following remarks.

Remark 1 (i) If $E$ is a vector space and $\mathcal{S}(\mathrm{E})$ is a linearly invariant family, then for every $x \in \mathrm{E}$, the set $\{\mathrm{x}\} \in \mathcal{S}(\mathrm{E})$.

(ii) The family $\mathcal{B}(\mathrm{G}, \mathcal{S}(\mathrm{E}))$ contains all constant functions.

Remark 2 Let $(\mathrm{G},+)$ be a group and $\mathrm{E}$ a vector space. Assume that $\mathcal{S}(\mathrm{E})$ is a linearly invariant family such that $\mathcal{B}(\mathrm{G}, \mathcal{S}(\mathrm{E}))$ satisfies the condition LIM or RIM. If $\mathrm{f}: \mathrm{G} \rightarrow \mathrm{E}$ is constant, then $\mathrm{M}[\mathrm{f}]=\operatorname{Im} \mathrm{f}$ (i.e., if $\mathrm{f}(\mathrm{x})=\mathrm{c}$ for $\mathrm{x} \in \mathrm{G}$, where $\mathrm{c} \in \mathrm{E}$, then $\mathrm{M}[\mathrm{f}]=\mathrm{c}$ ).

Remark 3 Let $\mathrm{G}$ be a group with metric $\mathrm{d}$ and let $\mathrm{E}$ be a normed space. Let $\mathrm{L} \in \mathbb{R}_{+}$, and

$$
\mathbf{d}(x, y):=\operatorname{Ld}(x, y) B(0,1),
$$

where $\mathrm{B}(0,1)$ is the closed ball with the center at 0 and the radius 1 . Then the function $\mathrm{f}: \mathrm{G} \rightarrow \mathrm{E}$ is Lipschitz with the constant $\mathrm{L}$ if and only if it is d-Lipschitz.

\section{Lipschitz approximation of Eq. (1)}

In this section, we can prove one of the main results of this paper.

Theorem 1 Let $\mathrm{G}$ be an Abelian group and $\mathrm{E}$ a vector space. Assume that $\mathcal{S}(\mathrm{E})$ is a linearly invariant family such that $\mathcal{B}(\mathrm{G}, \mathcal{S}(\mathrm{E}))$ admits LIM. Let $\mathrm{f}$ : $\mathrm{G} \rightarrow \mathrm{E}$ be an arbitrary function. If $\wedge \mathrm{f}(\cdot, \mathrm{y}): \mathrm{G} \rightarrow \mathrm{E}$ is $\mathbf{d}$-Lipschitz for every $\mathrm{y} \in \mathrm{G}$, then there exists a Drygas function $\mathrm{D}: \mathrm{G} \rightarrow \mathrm{E}$ such that $\mathrm{f}-\mathrm{D}$ is $\frac{1}{2} \mathbf{d}$ Lipschitz. Moreover, if $\operatorname{Im}(\Lambda \mathrm{f}) \subset \mathrm{V}$ for some $\mathrm{V} \in \mathcal{S}(\mathrm{E})$, then $\operatorname{Im}(\mathrm{f}-\mathrm{D}) \subset \frac{1}{2} \mathrm{~V}$. 
Proof. For every $a \in G$ we define $F_{a}: G \rightarrow E$ by

$$
F_{a}(y):=\frac{1}{2} f(a+y)+\frac{1}{2} f(a-y)-\frac{1}{2} f(y)-\frac{1}{2} f(-y), \quad y \in G .
$$

We will prove that $F_{a}$ belongs to $\mathcal{B}(G, \mathcal{S}(E))$. In fact, we have for $y, a \in G$,

$$
F_{a}(y)=\frac{1}{2} \wedge f(0, y)-\frac{1}{2} \wedge f(a, y)+f(a)-f(0) .
$$

So, $F_{a} \in \mathcal{B}(G, \mathcal{S}(E))$ for $a \in G$.

According to the assumptions, there exists a linear operator $M: \mathcal{B}(\mathrm{G}, \mathcal{S}(\mathrm{E})) \rightarrow$ E such that

(i) $\operatorname{Im}(\mathrm{g}) \subset \mathrm{V} \Rightarrow \mathrm{M}[\mathrm{g}] \in \mathrm{V}$,

(ii) if $g \in \mathcal{B}(G, \mathcal{S}(E))$ and $g_{a}: G \rightarrow E$ for $a \in G$ is defined by

$$
g_{a}(x):=g(a+x), \quad x \in G,
$$

then $g_{a} \in \mathcal{B}(G, \mathcal{S}(E))$ and $M\left[g_{a}\right]=M[g]$.

Consider the function $\mathrm{D}: \mathrm{G} \rightarrow \mathrm{E}$ given by

$$
\mathrm{D}(\mathrm{x}):=\mathrm{M}\left[\mathrm{F}_{\mathrm{x}}\right], \text { for } x \in \mathrm{G} \text {. }
$$

We will verify that $f-D$ is $\frac{1}{2} \mathbf{d}$-Lipschitz.

In view of our assumptions it follows that $\frac{1}{2} \wedge f(\cdot, y)$ is $\frac{1}{2} \mathbf{d}$-Lipschitz for every $y \in G$, which means that

$$
\frac{1}{2} \wedge f(x, y)-\frac{1}{2} \wedge f(z, y) \in \frac{1}{2} d(x, z)
$$

for all $x, z \in \mathrm{G}$. Let $\mathrm{l}: \mathrm{G} \rightarrow \mathrm{E}$ be the function

$$
l(x):=f(x)-M\left[F_{x}\right]=f(x)-D(x), \quad x \in G,
$$

and for any $x \in G, R_{x}: G \rightarrow E$ be defined by

$$
R_{x}(y):=f(x), \quad y \in G .
$$

Therefore, applying Remarks 1 and 2, one gets for all $x \in \mathrm{G}$,

$$
\begin{aligned}
l(x) & =f(x)-M\left[F_{x}\right]=M\left[R_{x}-F_{x}\right] \\
& =M\left[f(x)+\frac{1}{2} f(\cdot)+\frac{1}{2} f(-\cdot)-\frac{1}{2} f(x+\cdot)-\frac{1}{2} f(x-\cdot)\right] \\
& =M\left[\frac{1}{2} \Lambda f(x, \cdot)\right] .
\end{aligned}
$$


Immediately from (2) and (3) we obtain

$$
l(x)-l(z)=M\left[\frac{1}{2} \wedge f(x, \cdot)-\frac{1}{2} \wedge f(z, \cdot)\right], \quad x, z \in G .
$$

For any $x, z \in G$, we define $A_{(x, z)}: G \rightarrow E$ by

$$
A_{(x, z)}(y):=\frac{1}{2} \wedge f(x, y)-\frac{1}{2} \wedge f(z, y), \quad y \in G .
$$

By (2) we have $\operatorname{Im} A_{(x, z)} \subset \frac{1}{2} \mathbf{d}(x, z)$, which together with (4) implies

$$
l(x)-l(z)=M\left[A_{(x, z)}\right] \in \frac{1}{2} d(x, z),
$$

for all $y, z \in G$. This proves that

$$
(f(x)-D(x))-(f(z)-D(z)) \in \frac{1}{2} d(x, z), \text { for all } x, z \in G,
$$

i.e., $f-D$ is $\frac{1}{2} \mathbf{d}$-Lipschitz.

Now we will verify that D is a Drygas function. We have the equalities

$$
\begin{aligned}
D(x+z)+D(x-z) & =M\left[F_{x+z}(y)\right]+M\left[F_{x-z}(y)\right] \\
& =M\left[\frac{1}{2} f(x+z+y)+\frac{1}{2} f(x+z-y)-\frac{1}{2} f(y)-\frac{1}{2} f(-y)\right] \\
& =M\left[\frac{1}{2} f(x-z+y)+\frac{1}{2} f(x-z-y)-\frac{1}{2} f(y)-\frac{1}{2} f(-y)\right]
\end{aligned}
$$

and

$$
\begin{aligned}
2 D(x)+D & (z)+D(-z)=2 M\left[F_{x}(y)\right]+M\left[F_{z}(y)\right]+M\left[F_{-z}(y)\right] \\
= & 2 M\left[\frac{1}{2} f(x+y)+\frac{1}{2} f(x-y)-\frac{1}{2} f(y)-\frac{1}{2} f(-y)\right] \\
& +M\left[\frac{1}{2} f(z+y)+\frac{1}{2} f(z-y)-\frac{1}{2} f(y)-\frac{1}{2} f(-y)\right] \\
& +M\left[\frac{1}{2} f(-z+y)+\frac{1}{2} f(-z-y)-\frac{1}{2} f(y)-\frac{1}{2} f(-y)\right] \\
= & M\left[\frac{1}{2} f(x+y+z)+\frac{1}{2} f(x-y+z)-\frac{1}{2} f(y+z)-\frac{1}{2} f(-y+z)\right] \\
& +M\left[\frac{1}{2} f(x+y-z)+\frac{1}{2} f(x-y-z)-\frac{1}{2} f(y-z)-\frac{1}{2} f(-y-z)\right] \\
& +M\left[\frac{1}{2} f(z+y)+\frac{1}{2} f(z-y)-\frac{1}{2} f(y)-\frac{1}{2} f(-y)\right]
\end{aligned}
$$




$$
\begin{aligned}
& +M\left[\frac{1}{2} f(-z+y)+\frac{1}{2} f(-z-y)-\frac{1}{2} f(y)-\frac{1}{2} f(-y)\right] \\
= & D(x+z)+D(x-z) .
\end{aligned}
$$

It follows that D is a Drygas function.

To finish the proof assume that $\operatorname{Im}(\Lambda f) \subset \mathrm{V}$ for some $\mathrm{V} \in \mathcal{S}(\mathrm{E})$. Then we have $\operatorname{Im}\left(\frac{1}{2} \wedge f\right) \subset \frac{1}{2} \vee$. In view of (3) we get $f(x)-D(x)=M\left[\frac{1}{2} \wedge f(x, \cdot)\right] \in$ $\frac{1}{2} V$ for all $x \in G$. Thus $\operatorname{Im}(f-D) \subset \frac{1}{2} V$, which completes the proof of the theorem.

Corollary 1 Let $\mathrm{G}$ be an Abelian group and $(\mathrm{E},\|\cdot\|)$ a normed space. Assume that $\mathcal{S}(\mathrm{E})$ is a family of closed balls such that $\mathcal{B}(\mathrm{G}, \mathcal{S}(\mathrm{E})$ ) admits LIM. Let $\mathrm{f}: \mathrm{G} \rightarrow \mathrm{E}$ and $\mathrm{g}: \mathrm{G} \rightarrow \mathbb{R}_{+}$satisfy the inequality

$$
\|\wedge f(x, y)-\Lambda f(z, y)\| \leq g(x-z)
$$

for all $\mathrm{x}, \mathrm{y}, z \in \mathrm{G}$. Then there exists a Drygas function $\mathrm{D}: \mathrm{G} \rightarrow \mathrm{E}$ such that

$$
\|(f-D)(x)-(f-D)(y)\| \leq(1 / 2) g(x-y)
$$

for all $x, y \in G$, where $(f-D)(x) \equiv f(x)-D(x)$.

Proof. We put

$$
\mathrm{d}(x, y):=\mathrm{g}(x-\mathrm{y}) \mathrm{B}(0,1), \quad x, y \in \mathrm{G}
$$

where $\mathrm{B}(0,1)$ is the closed unit ball with center at zero. By (5) we obtain

$$
\wedge f(x, y)-\Lambda f(z, y) \in \mathbf{d}(x, z), \quad x, y, z \in G,
$$

which means that $\Lambda f(\cdot, y)$ is a d-Lipschitz. Therefore, from Theorem 1 there exists a Drygas function $D: G \rightarrow E$ such that $f-D$ is $(1 / 2) d$-Lipschitz. By the definition of $\mathbf{d}$ we get the desired result.

\section{Approximation with Lipschitz norm}

We shall introduce the following definition (see also [20]).

Definition 6 A group $(\mathrm{G},+, \mathrm{d}, \widetilde{\mathrm{d}})$ is said to be a metric pair if

(1) $(\mathrm{G},+, \mathrm{d})$ is an Abelian metric group,

(2) $\widetilde{\mathrm{d}}:(\mathrm{G} \times \mathrm{G}) \times(\mathrm{G} \times \mathrm{G}) \rightarrow \mathbb{R}_{+}$is a metric in $\mathrm{G} \times \mathrm{G}$, 
(3) $\widetilde{d}((a, x),(a, y))=\widetilde{d}((x, a),(y, a))=d(x, y)$ for $x, y, a \in G$.

The following lemma is needed to establish the next results.

Lemma 1 Let $(\mathrm{G},+, \mathrm{d}, \widetilde{\mathrm{d}})$ be a metric pair and $(\mathrm{E},\|\cdot\|)$ a normed space. Assume that $\mathcal{S}(\mathrm{E})$ is a family of closed balls such that $\mathcal{B}(\mathrm{G}, \mathcal{S}(\mathrm{E}))$ admits LIM. Let $\mathrm{f}: \mathrm{G} \rightarrow \mathrm{E}$ be a function and $w: \mathbb{R}_{+} \rightarrow \mathbb{R}_{+}$be the module of continuity of the function $\Lambda \mathrm{f}: \mathrm{G} \times \mathrm{G} \rightarrow \mathrm{E}$. Then there exists a Drygas function $\mathrm{D}: \mathrm{G} \rightarrow \mathrm{E}$ such that the function $(1 / 2) w$ is the module of continuity of $\mathrm{f}-\mathrm{D}$. Moreover, if $\wedge f \in \mathcal{B}(\mathrm{G} \times \mathrm{G}, \mathcal{S}(\mathrm{E}))$, then

$$
\|f-D\|_{\text {sup }} \leq(1 / 2)\|\wedge f\|_{\text {sup }}
$$

Proof. Define $\mathbf{d}: \mathrm{G} \times \mathrm{G} \rightarrow \mathcal{S}(\mathrm{E})$ by the formula

$$
d(x, y):=\left(\inf _{t \geq d(x, y)} w(t)\right) B(0,1),
$$

where $\mathrm{B}(0,1)$ is the closed unit ball with center at zero. Since $w$ is the module of continuity of $\Lambda f(\cdot, y)$ for $y \in G$, we have

$$
\|\wedge f(x, y)-\Lambda f(z, y)\| \leq \inf _{t \geq \widetilde{d}((x, y),(z, y))} w(t), \quad x, y, z \in G .
$$

This implies that

$$
\|\wedge f(x, y)-\wedge f(z, y)\| \leq \inf _{t \geq d(x, z)} w(t), \quad x, y, z \in G
$$

i.e.,

$$
\wedge f(x, y)-\Lambda f(z, y) \in d(x, z), \quad \text { for } x, y, z \in G .
$$

This shows that $\mathrm{Qf}(\cdot, \mathrm{y})$ is $\mathbf{d}$-Lipschitz.

Now, in view of Theorem 1, there exists a Drygas function $D: G \rightarrow E$ such that $f-D$ is $(1 / 2) \mathbf{d}$-Lipschitz and consequently

$$
(f(x)-D(x))-(f(y)-D(y)) \in(1 / 2) d(x, y), \quad x, y \in G .
$$

This is equivalent to the condition

$$
\|(f(x)-D(x))-(f(y)-D(y))\| \leq \inf _{t \geq d(x, y)}(1 / 2) w(t), \quad x, y \in G .
$$

This shows that $(1 / 2) w$ is the module of continuity of $f-D$. 
Finally, assume that $\Lambda f \in \mathcal{B}(G \times G, \mathcal{S}(E))$. Thus the following set is well defined:

$$
W:=B\left(0,\|\Lambda f\|_{\text {sup }}\right) \quad \text { with } \operatorname{Im}(\Lambda f) \subset W .
$$

Thus from Theorem 1, we get

$$
\operatorname{Im}(f-D) \subset(1 / 2) W
$$

which implies the inequality (7) and completes the proof.

In the remaining part of the paper, we investigate two results about the stability of the generalized quadratic functional equation in the Lipschitz norms.

Theorem 2 Let $(\mathrm{G},+, \mathrm{d}, \widetilde{\mathrm{d}})$ be a metric pair and $(\mathrm{E},\|\cdot\|)$ a normed space. Assume that $\mathcal{S}(\mathrm{E})$ is a family of closed balls such that $\mathcal{B}(\mathrm{G}, \mathcal{S}(\mathrm{E}))$ admits LIM.

(i) Let $\mathrm{f}: \mathrm{G} \rightarrow \mathrm{E}$ be a function satisfying the condition $\wedge \mathrm{f} \in \operatorname{Lip}(\mathrm{G} \times \mathrm{G}, \mathrm{E})$. Then there exists a Drygas function $\mathrm{D}: \mathrm{G} \rightarrow \mathrm{E}$ such that

$$
\|f-D\|_{\text {Lip }} \leq(1 / 2)\|\wedge f\|_{\text {Lip }}
$$

(ii) Let $\mathrm{f}: \mathrm{G} \rightarrow \mathrm{E}$ be a function satisfying the condition $\Lambda \mathrm{f} \in \operatorname{Lip}^{0}(\mathrm{G} \times \mathrm{G}, \mathrm{E})$. Then there exists a Drygas function $\mathrm{D}: \mathrm{G} \rightarrow \mathrm{E}$ such that

$$
\|f-D\|_{\operatorname{Lip}^{0}} \leq(1 / 2)\|\Lambda f\|_{\operatorname{Lip}^{0}} .
$$

Proof. (i) Consider $w: \mathbb{R}_{+} \rightarrow \mathbb{R}_{+}$by the formula

$$
w(x):=\operatorname{lip}(\Lambda f) x, \quad \text { for } x \in \mathbb{R}_{+} .
$$

Since $\Lambda f \in \operatorname{Lip}(G \times G, E)$, we obtain

$$
\begin{aligned}
\|\wedge f(x, y)-\Lambda f(t, z)\| & \leq \operatorname{lip}(\Lambda f) \widetilde{d}((x, y),(t, z)) \\
& =w(\widetilde{d}((x, y),(t, z))), \quad x, y, t, z \in G,
\end{aligned}
$$

which means that $w$ is the module of continuity of $\Lambda f$. Thus, by Lemma 1 , there exists a Drygas function $D: G \rightarrow E$ such that $(1 / 2) w$ is the module of continuity of $f-D$. Thus we have the inequality

$$
\begin{aligned}
\|(f(x)-D(x))-(f(y)-D(y))\| & \leq(1 / 2) w(d(x, y)) \\
& =(1 / 2) \operatorname{lip}(\Lambda f) d(x, y), \quad x, y \in G .
\end{aligned}
$$


This inequality implies that $f-D$ is a Lipschitz function and

$$
\operatorname{lip}(f-D) \leq \frac{1}{2} \operatorname{lip}(\wedge f) .
$$

Taking into account that $\Lambda f \in \operatorname{Lip}(\mathrm{G} \times \mathrm{G}, \mathrm{E})$, we have also $\wedge f \in \mathcal{B}(\mathrm{G} \times \mathrm{G}, \mathcal{S}(\mathrm{E}))$. Therefore by Lemma 1 we obtain

$$
\|\mathrm{f}-\mathrm{D}\|_{\text {sup }} \leq \frac{1}{2}\|\wedge \mathrm{f}\|_{\text {sup }},
$$

that is, $f-D \in \operatorname{Lip}(G, E)$. Finally, from (12) and (13), we obtain the desired result.

(ii) By the same reasoning as in the proof of (i) we can prove that there exists a Drygas function $D: G \rightarrow E$ such that $f-D$ is Lipschitz and

$$
\operatorname{lip}(f-D) \leq \frac{1}{2} \operatorname{lip}(\wedge f) .
$$

Since $\mathrm{D}(0)=0$, we obtain

$$
\|f(0)-D(0)\|=\|f(0)\|=(1 / 2)\|\Lambda f(0,0)\| .
$$

Thus

$$
\begin{aligned}
\|f-D\|_{\operatorname{Lip}^{0}} & =\|f(0)-D(0)\|+\operatorname{lip}(f-D) \\
& \leq \frac{1}{2}\|\wedge f(0,0)\|+\frac{1}{2} \operatorname{lip}(\wedge f) \\
& =\frac{1}{2}\|\wedge f\|_{\operatorname{Lip}^{0}}
\end{aligned}
$$

which completes the proof.

\section{References}

[1] T. Aoki, On the stability of the linear transformation in Banach spaces, J. Math. Soc. Japan, 2 (1950), 64-66.

[2] R. Badora, On some generalized invariant means and their application to the stability of the Hyers-Ulam type, Ann. Polon. Math., 58 (1993), $147-159$.

[3] S. Czerwik, K. Dlutek, Stability of the quadratic functional equation in Lipschitz spaces, J. Math. Anal. Appl., 293 (2004), 79-88. 
[4] H. Drygas, Quasi-inner products and their applications, in Advances in Multivariate Statistical Analysis, K. Gupta, Ed., Reidel (1987), 13-30.

[5] B. R. Ebanks, P. I. Kannappan, P. K. Sahoo, A common generalization of functional equations characterizing normed and quasi-inner-product spaces, Can. Math. Bull., 35 (1992), 321-327.

[6] Iz. EL-Fassi, Generalized hyperstability of a Drygas functional equation on a restricted domain using Brzdek's fixed point theorem, J. Fixed Point Theory Appl., 19 (4) (2017), 2529-2540.

[7] Iz. EL-Fassi, On approximation of approximately generalized quadratic functional equation via Lipschitz criteria, Quaest. Math., 42 (5) (2019), $651-663$.

[8] Iz. EL-Fassi, A. Chahbi, S. Kabbaj, Lipschitz stability of the k-quadratic functional equation, Quaest. Math., 40 (8) (2017), 991-1001.

[9] G. L. Forti, Hyers-Ulam stability of functional equations in several variables, Aequationes Math., 50 (1995), 143-190.

[10] G. L. Forti, J. Sikorska, Variations on the Drygas equation and its stability, Nonlinear Anal., 74 (2011), 343-350.

[11] Z. Gajda, On stability of additive mappings, Int. J. Math. Math. Sci., 14 (1991), 431-434.

[12] Z. Gajda, Invariant Means and Representations of Semi-groups in the Theory of Functional Equations, Prace Naukowe Uniwersytetu Ślaskiego, Katowice, 1992.

[13] F. P. Greenleaf, Invariant Means on Topological Groups and Their Applications, in: Van Nostrand Mathematical Studies, vol. 16, Van Nostrand, New York, 1969.

[14] D. H. Hyers, On the stability of the linear functional equation, Proc. Nat. Acad. Sci. USA, 27 (1941), 222-224.

[15] D. H. Hyers, G. Isac, Th. M. Rassias, Stability of Functional Equations in Several Variables, Birkhäuser, Boston, 1998.

[16] S.-M. Jung, P. K. Sahoo, Stability of a functional equation of Drygas, Aequationes Math., 64 (2002), 263-273. 
[17] Th. M. Rassias, On the stability of the linear mapping in Banach spaces, Proc. Amer. Math. Soc., 72 (1978), 297-300.

[18] P. K. Sahoo, P. Kannappan, Introduction to functional equations, $C R C$ Press, Boca Raton, Fla, USA (2011).

[19] J. Sikorska, On a direct method for proving the Hyers-Ulam stability of functional equations, J. Math. Anal. Appl., 372 (2010), 99-109.

[20] J. Tabor, Lipschitz stability of the Cauchy and Jensen equations, Results Math., 32 (1997), 133-144.

[21] S. M. Ulam, A Collection of Mathematical Problems, Interscience, New York, 1960.

[22] D. Yang, Remarks on the stability of Drygas equation and the Pexiderquadratic equation, Aequat. Math., 68 (2004), 108-116.

Received: March 14, 2018 